\title{
GPS and GSM based Passenger Tracking System
}

\author{
Dalip \\ Department of Information Technology, \\ MMEC, Maharishi Markandeshwar University, \\ Mullana, Haryana, India
}

\author{
Vijay Kumar, Ph.D \\ Department of Computer Science and Engineering, \\ MMEC, Maharishi Markandeshwar University, \\ Mullana, Haryana, India
}

\begin{abstract}
Evolution in transportation technology makes the necessity for increasing passenger safety. Today women safety is more important when they are travelling in public transport. So we want a tracking system for passengers so that we can monitor at anytime from anywhere. Mostly passenger tracking systems are Radio Frequency Identification (RFID) based but they are not cost effective. In this paper a cost effective Global Positioning System (GPS) and Global System for Mobile communication (GSM) based passengers tracking system inside buses is introduced here. It tracks the passengers by using ticket number and displays location on Google map.
\end{abstract}

\section{Keywords}

GPS, GSM, SMS, Ticket Number, Google map.

\section{INTRODUCTION}

Mostly vehicle tracking systems are based on GPS and GSM. Short Messaging Service (SMS) [1] is a feature available on all mobile phones which allows a small amount of text to be sent between one user and another. GPS consists of a [3] network of 24 satellites in six different 12-hour orbital paths spaced so that at least five are in view from every point on the globe. Today, GPS has a [2] [3] wide range of other applications including tracking package delivery, mobile commerce, emergency response, exploration, surveying, law enforcement, recreation, wildlife tracking, search and rescue, roadside assistance, stolen vehicle recovery, satellite data processing, and resource management. CELL PHONES become [10] more ubiquitous in our daily lives, the need for context-aware applications increases. One of the main context information is location, which enables a wide set of cell phone applications including navigation, location-aware social networking, and security. Automatic vehicle tracking (AVL) is a system [6] that provides the tracking of vehicle. Location Based Service (LBS), Navigation and Intelligent Transportation are the hot research topics now. Daily millions of passengers travel in buses to reach his/her destination. A major concern is the family members who wish to monitor to each other when they are moving in buses. For passengers safety point of view a passenger tracking system is required. Which can track of passengers between source and destination? Mostly passenger tracking system use RFID tags for tracking. But these tracking systems are not cost effective. So a cost effective system is introduced here by using GSM and GPS, which track passengers those are inside buses. It tracks passengers on the basis of bus ticket number. Only users need to enter the valid ticket number of passengers. Users of the system can track passengers at anytime and anywhere by using mobile phone and laptop/desktop. The users of this system can get location information of passenger, only if they know ticket number. For this tracking system its duty of passengers to tell your ticket number to his/her known and family members before starting journey.

The rest of paper explained as follow. The section II described the related work for GPS and GSM based passenger tracking system. Section III shows proposed system. The section IV describes the conclusion of the paper.

\section{RELATED WORK}

In urban traffic problems [7] and city planning problems combines the General Packet Radio Service (GPRS) and Bluetooth technology, realizing the intelligence in some aspects just like the management of bus passengers in modern city, the data collection of passengers in the bus, the management of the driver, the GPS location of the buses, the formulating plans, the real-time monitoring of bus, the inquiring information and the statistical analysis about the passengers, the services of electronic [4] platform. An Automatic Vehicle Location System (AVLS) is a system [8] which takes the advantages of new advancement in both wireless communications as well as mobile computations. System uses Global Positioning Systems (GPS) and Geospatial Information System (GIS) data to locate vehicles and get additional information about the location and features of the vehicle location [5]. It composed of there main features: 1) positioning, 2) data transfer and 3) data visualization. Prototype of AVLS is based on widely accessible technologies. An AVLS uses GPS for localization of users and Pocket PC for communication with data servers. Since other wireless technologies like GPRS has not been established very well in Iran, therefore, it is decided to design a GSM based system. It integrates GPS installation [6] in vehicles, data transmission through wireless network services (e.g. SMS, GPRS); direction promotions supported by the GIS based way-finding algorithms; and Web real-time video capture systems. This methodology gives us the possibility to solve total Location Based Service (LBS), Intelligent and Navigation Transportation questions. Multi Agent System (MAS) [14] architecture implementation used to solve these two important problems in the Web integration of the GPS+GIS+GPRS Tracking System and Real-time Monitoring System. The transit information [11] on an Open Street Map (OSM) web interface and sends this information on the Google Android mobile device. The content is in the form of predicted arrival and departure times for buses at userselectable geographic locations within a transit region. This application uses the real-time information such as current location and timestamp of both bus and users to provide bus route information. The public interface of system provides graphical views which used to updates and display the vehicle locations and to allow users to see routes, stops and moving buses. This system helps in case of traffic speed violation, a GPRS message [16] containing information about the vehicle such as location and maximum speed is sent to a hosting server located in an authorized office so that the violated 
vehicle is ticketed. It can also track the vehicle's current location on a Google Map, which is mostly beneficial when vehicles should follow a specific road and in case of robbery. Some sensors attached to the air-bags in vehicles, are attached to the system that in case of accident, it will send notifications to the civil defense, nearest hospital and police station. An efficient automotive [15] security system is implemented for anti-theft using an embedded system occupied with a GPS and a GSM. The client interacts through this system with vehicles and determines their current locations and status using Google Earth. The user can track the position of targeted vehicles on Google Earth. Coordinates of GPS are corrected using a discrete Kalman filter. An integrated GPS-GSM system [12] also use Kalman filter to enhance the accuracy of measured position. After data processing, Google Earth application is used to view the current location and status of each vehicle. This objective of this system is to manage fleet, police automobiles distribution and car theft cautions. Due to some criminal activities Parents always worry about the possibility of kidnapping of their children. Android based solution [17] to aid parents to track their children in real time. It takes the advantage of the location services provided by mobile phone since most of kids carry mobile phones. The mobile application use the GPS and SMS services found in Android mobile phones.

Some other several authors [13] [9] also have described GPS and GSM based vehicle tracking system.

\section{APPLICATION DEVELOPMENT}

The application is designed for passengers and who want to track passengers. Both must have mobile phones that Support GPRS and SMS. The application is mostly to be used passengers tracking users to track down the passenger's location. This section provides a more detailed description of the overall system and functionality of the main components of the proposed architecture. The system modal is designed in a way so that it provides the maximum accessibility for the users at anytime from anywhere by providing two types of end user applications, a mobile application and web application. In figure 1 GPS and GSM device are placed on buses. GPS device receives coordinate information from satellites. GPS device is also connected with GSM device and send the GPS coordinate information using SMS to administrator control area with the help of GSM tower. The system consists of two sides, passengers and tracking user's side. A tracking users device main duty is to send a request ticket number SMS to the server to get the location of the passenger. On the other hand, the passenger's device main responsibility is to reply the ticket number to his/her known before starting journey. When the users request location from the mobile and web application after registering and logging into web server, web server response corresponding user's request.

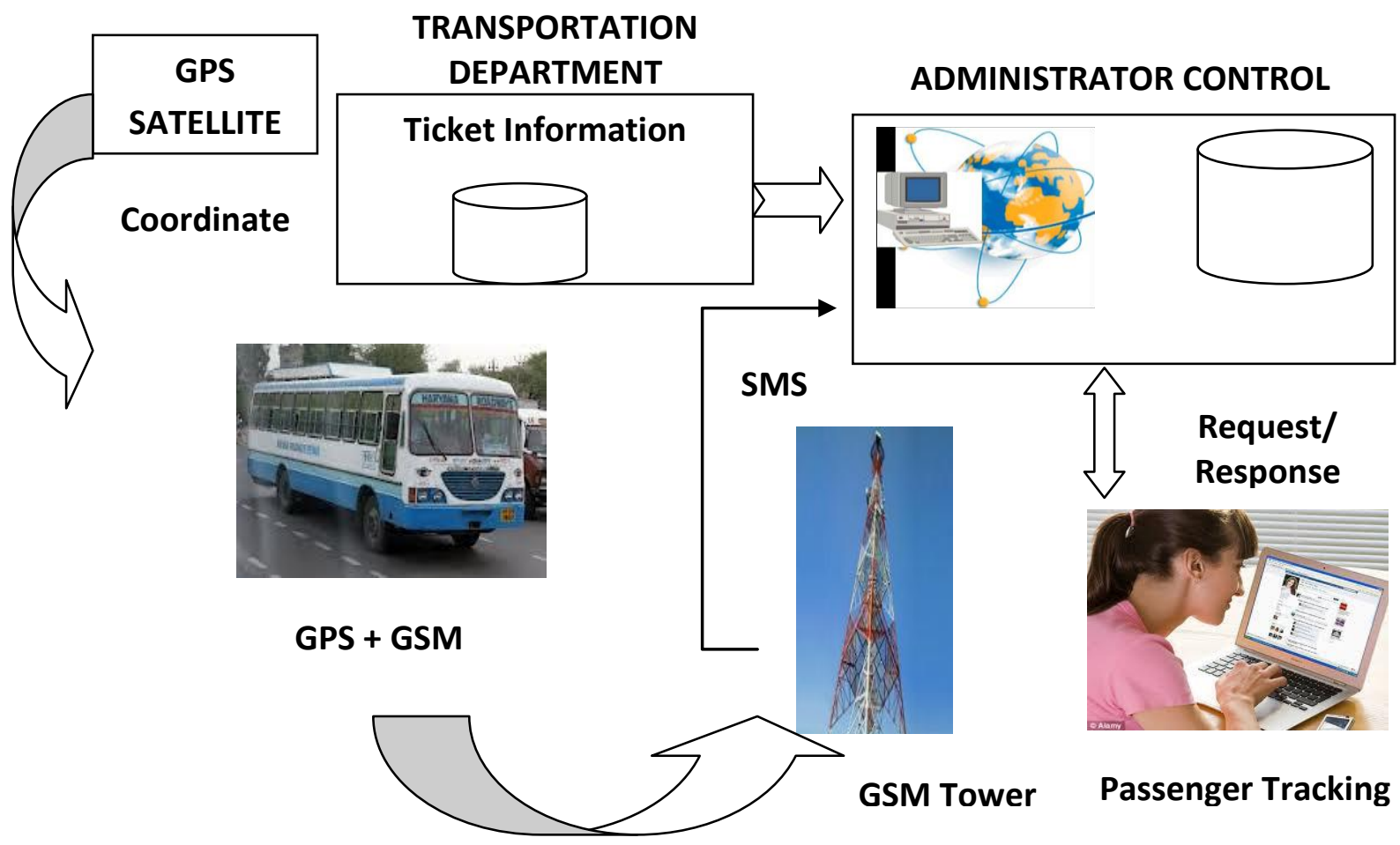

Figure1: Passenger Tracking System

All vehicle navigation and ticket number information is stored in database. Ticket number is unique ID for all tracked passengers. Server and database are connected to each other. For passengers tracking history of vehicle movement and information of ticket number are required. Vehicle movement history will store automatically threw SMS and ticket number information will store manually by transportation department at server side. During the time of issuing ticket to passengers this information is stored by transportation department in data base corresponding to the particular buses ID and route. The input for the system is ticket number. Users of this system can send query with the help of SMS and web browser. After received users query as a ticket number server process the the history of passengers as starting journey time, journey date, ending journey time, last status and current status as shown in table 5 . 


\subsection{DATABASE}

From the client point of view data can be stored locally or acquired from the server. Data can be hold in a database, sent by devices or transferred from other data servers. Overview of database is given below. Table 1 shows route wise history of vehicle navigation. This information is obtained by using GPS and GSM devices. The values of latitude, longitude, data, time and speed parameters are sent by SMS to server using GSM modem. Server automatically stores this information into database. Table 2, table 3 and table 4 shows route wise ticket number corresponding to bus ID. Table 5 contains the history of passenger navigation. Every ticket number represents unique passengers inside buses.

Table1: History of vehicle navigation.

\begin{tabular}{|c|c|c|c|c|c|c|c|c|}
\hline Bus ID & Route & Bus Stops & Latitude & Longitude & Date & Time & $\begin{array}{l}\text { Distance } \\
\text { Covered }\end{array}$ & Speed \\
\hline HR001AB & YnrtoDelhi & Radur & 30.0320932 & 77.1485051 & $1-6-2014$ & 5:08AM & $23 \mathrm{Km}$ & $45 \mathrm{~km} / \mathrm{hr}$ \\
\hline HR002AB & YnrtoDelhi & Ladwa & 29.9955086 & 77.0488128 & $1-6-2014$ & $7: 30 \mathrm{PM}$ & $30 \mathrm{Km}$ & $50 \mathrm{~km} / \mathrm{hr}$ \\
\hline HR003AB & YnrtoDelhi & Karnal & 29.7192979 & 76.9819346 & $1-6-2014$ & 9:00AM & $4 \mathrm{Km}$ & $55 \mathrm{~km} / \mathrm{hr}$ \\
\hline HR011CD & AmbtoChd & Ambala & 29.6797275 & 76.9904825 & $2-6-2014$ & $2: 00 \mathrm{PM}$ & $15 \mathrm{Km}$ & $40 \mathrm{~km} / \mathrm{hr}$ \\
\hline HR022CD & AmbtoChd & Chandigarh & 29.6797275 & 76.9904825 & $3-5-2014$ & 3:40PM & $30 \mathrm{Km}$ & $75 \mathrm{~km} / \mathrm{hr}$ \\
\hline HR033EF & PantoDelhi & Sonipat & 28.9243676 & 77.1011065 & $5-5-2014$ & $8: 35 \mathrm{AM}$ & $45 \mathrm{Km}$ & $64 \mathrm{~km} / \mathrm{hr}$ \\
\hline HR044EF & PantoDelhi & Delhi & 28.9243676 & 77.1011065 & $8-5-2014$ & 9:10AM & $28 \mathrm{Km}$ & $70 \mathrm{~km} / \mathrm{hr}$ \\
\hline
\end{tabular}

Table 2: Route1 (YnrtoDehli)

\begin{tabular}{|c|c|}
\hline Bus ID & Ticket No. \\
\hline HR001AB & HR0986345AS \\
\hline HR001AB & HR0986346AS \\
\hline HR001AB & HR0986347AS \\
\hline HR002AB & HR0996335AS \\
\hline HR002AB & HR0996336AS \\
\hline HR002AB & HR0996337AS \\
\hline
\end{tabular}

Table3: Route2: (AmbtoChd)

\begin{tabular}{|c|c|}
\hline Bus ID & Ticket No. \\
\hline HR011CD & HR0886355AF \\
\hline HR011 CD & HR0886355AF \\
\hline HR011 CD & HR0886355AF \\
\hline HR022 CD & HR0876355AF \\
\hline HR022 CD & HR0876355AF \\
\hline HR022 CD & HR0876355AF \\
\hline
\end{tabular}

Table4: Route3(PantoDelhi)

\begin{tabular}{|c|c|}
\hline Bus ID & Ticket No. \\
\hline HR033EF & HR0386325AG \\
\hline HR033 EF & HR0386326AG \\
\hline HR033 EF & HR0386327AG \\
\hline HR044 EF & HR0486317AG \\
\hline HR044 EF & HR0486318AG \\
\hline HR044 EF & HR0486319AG \\
\hline
\end{tabular}

Table 5: History of Passenger Navigation

\begin{tabular}{|c|c|c|c|c|c|c|c|}
\hline Bus ID & Route & Ticket No. & $\begin{array}{c}\text { Journey } \\
\text { Date }\end{array}$ & $\begin{array}{c}\text { Starting } \\
\text { Journey } \\
\text { Time }\end{array}$ & $\begin{array}{c}\text { Ending } \\
\text { Journey } \\
\text { Time }\end{array}$ & $\begin{array}{c}\text { Last } \\
\text { Status of } \\
\text { Passenger }\end{array}$ & $\begin{array}{c}\text { Current } \\
\text { Status of } \\
\text { Passenger }\end{array}$ \\
\hline HR001AB & YnrtoDelhi & HR0986345AS & $1-6-2014$ & $8: 30$ AM & $12: 30$ PM & Ladwa & Panipat \\
\hline HR002AB & YnrtoDelhi & HR0996335AS & $1-6-2014$ & $6: 00$ AM & $10: 00$ AM & Radaur & Sonipat \\
\hline HR003AB & YnrtoDelhi & HR0996337AS & $1-6-2014$ & $1: 20$ PM & $5: 30$ PM & Indri & Karnal \\
\hline HR011CD & AmbtoChd & HR0886355AF & $2-6-2014$ & $10: 10$ AM & $1: 00$ PM & Ambala & Zirakpur \\
\hline HR022CD & AmbtoChd & HR0876355AF & $3-5-2014$ & $4: 00$ PM & $7: 00$ PM & Zirakpur & Chandigarh \\
\hline HR033EF & PantoDelhi & HR0386325AG & $5-5-2014$ & $3: 00$ PM & $6: 00$ PM & Sonipat & Delhi \\
\hline HR044EF & PantoDelhi & HR0486319AG & $8-5-2014$ & $2: 00$ PM & $4: 00$ PM & Panipat & Delhi ByPass \\
\hline
\end{tabular}

\subsection{RESULTS AND DISCUSSION}

This section presents the selected results of our experiments. The purpose of these experiments is show a functional prototype of proposed system. We mounted Bluetooth based GPS, which is connected to mobile phone into private buses. Mobile phone send tracking data at server by SMS. Laptop is used to track the passenger on Google map. 


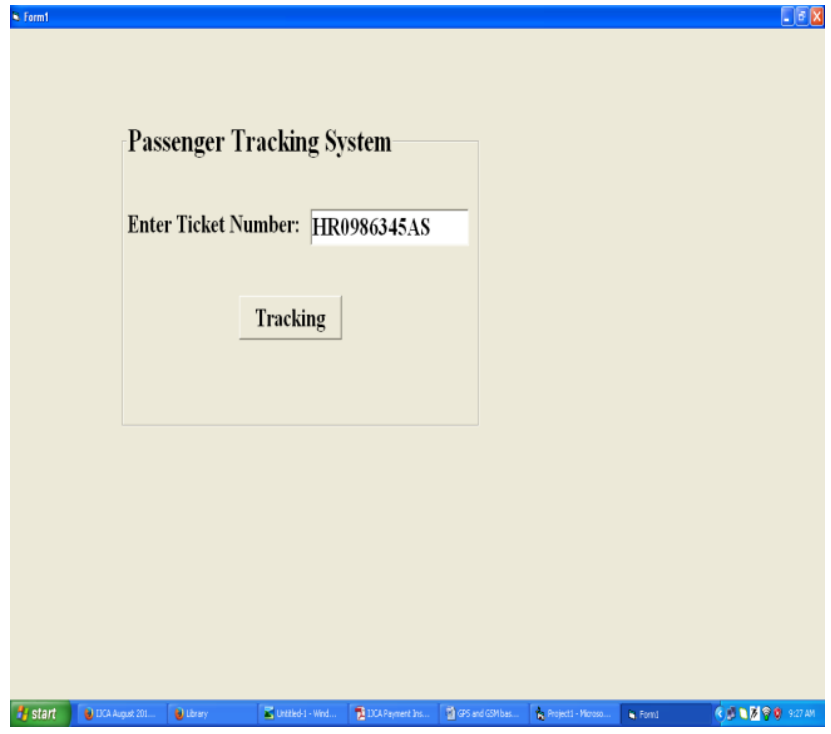

Figure 2: Tracking with Ticket Number

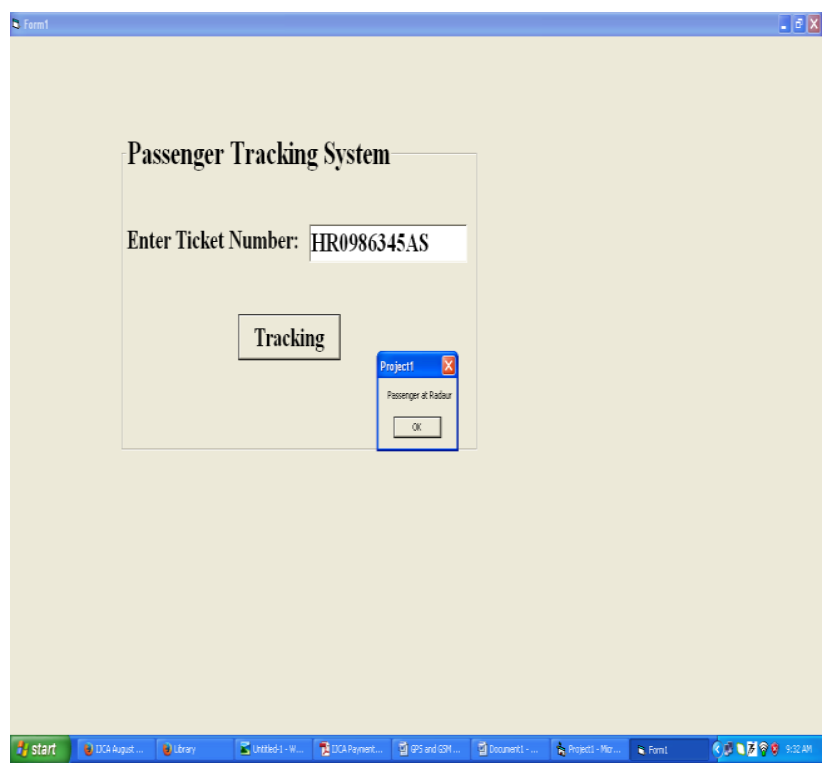

Figure3: Display Passenger Tracking Information

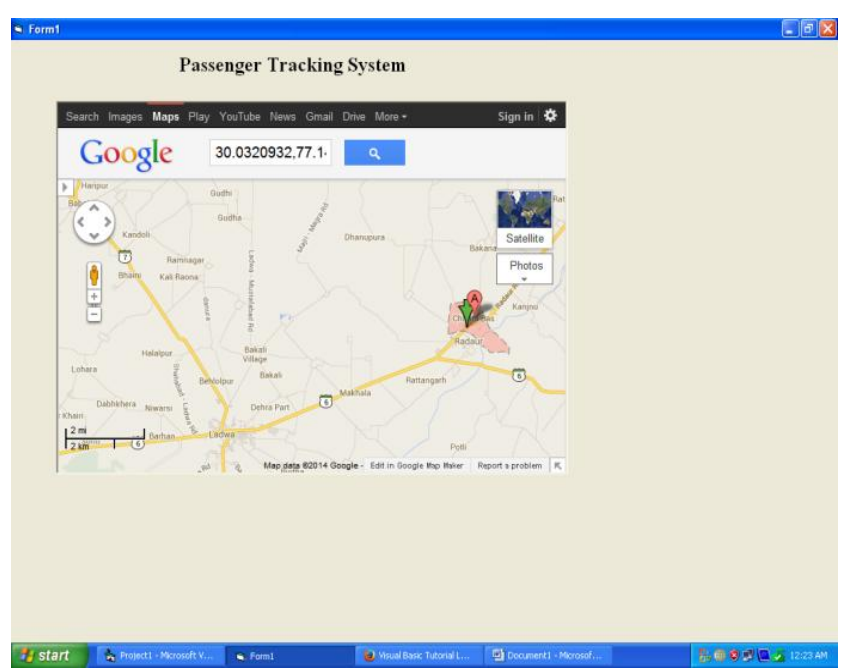

Figure 4: Display Passenger Location on Google Map [18]

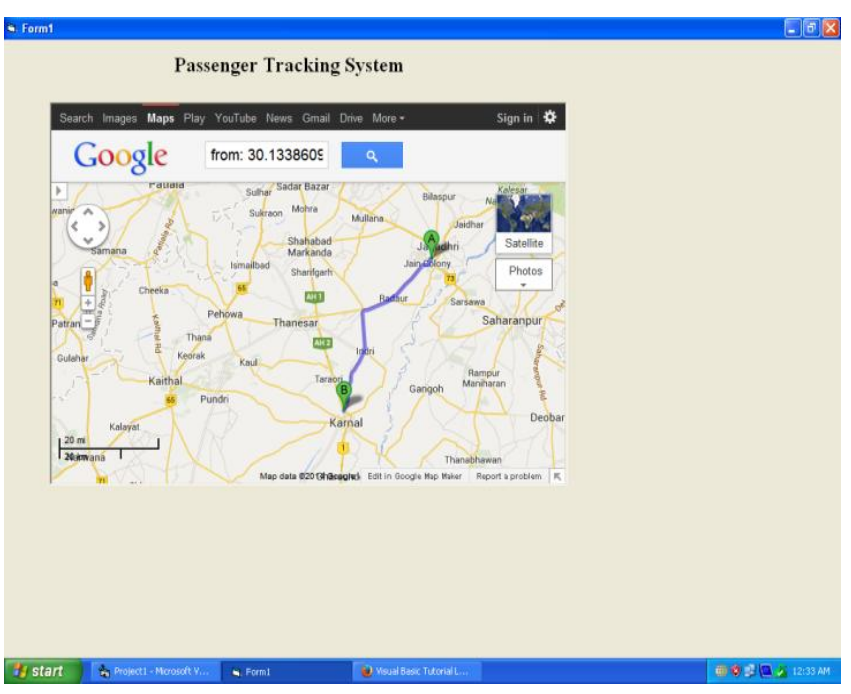

Figure 5: Distance covered by Passenger on Google Map [18]

The input for the system is ticket number as shown in figure 2. In figure 3 after enter valid ticket number it shows passenger tracking information. It also displays the location on Google map. Figure 4 shows passenger at Radaur city on Google map. Figure 5 represents how much distance is covered by passenger. The proposed system is cost effective because no any other costly devices are required for tracking, except Bluetooth enable GPS receiver and GSM mobile phone.

\section{CONCLUSION}

In conclusion, this project was developed to aid locating passengers. Proposed passenger tracking system is efficient in term of system cost. This system tracks the passengers on the basis of vehicle history and ticket number. It provides the history of passenger movement and displays location on Google map. Tracking is easy for the users of this system because they just need to enter the valid ticket number only. Features can be added to enhance the system such as Geofencing, emergency alerts and many others.

\section{REFERENCES}

[1] SMS Quick Start Guide, "Changemakers Ashoka innovators for the public", Arilington, VA 22209-1929 USA. www.changemakers.com

[2] P. Enge and P. Misra, "Special issue on GPS: The global positioning system," Proc. IEEE, vol. 87, no. 1, pp. 3-15, Jan. 1999.

[3] Rashmi Bajaj, Samantha Lalinda Ranaweera and Dharma P. Agrawa," GPS: Location Tracking Technology", Communication, April 2002.

[4] Andrei Gurtov, Matti Passoja, Olli Aalto And Mike Raitola,Multi-Layer Protocol Tracing in a GPRS Network ,IEEE Fall VTC 2004: 124-130.

[5] Shah, D., Patel, D., Chaudhary and S," Grid-Based Vehicle Locating System", pp. 53-67. Springer, Heidelberg (2006).

[6] Ye Lei and Lin Hui," The Web Integration of the GPS+GPRS+GIS Tracking System and Real-Time Monitoring System Based on MAS ", J.D. Carswell and T. Tezuka (Eds.): W2GIS, LNCS 4295, pp. $54-65$, ( Springer-Verlag Berlin Heidelberg 2006.

[7] Song Jie, Li Na-na, Chen Ji-lin, Dong Yong-feng and Zhao Zheng," Design and Implementation of Intelligent Transportation System Based on GPRS and Bluetooth 
hybrid model ",Proceedings of the IEEE International Conference on Automation and Logistics Qingdao, China September 2008.

[8] Ali Mousavi, Mohammad A. Rajabi, and Mohammad Akbari, "Design and Implementation of a GSM Based Automatic Vehicle Location System ",ICCSA, Part I, LNCS 5592, pp. 533-542, () Springer-Verlag Berlin Heidelberg 2009.

[9] Khondker Shajadul Hasan, Mashiur Rahman, Abul L. Haque, M Abdur Rahman, Tanzil Rahman and M Mahbubur Rasheed," Cost Effective GPS-GPRS Based Object Tracking System", Proceedings of the International MultiConference of Engineers and Computer Scientists Vol I IMECS 2009, March 18 - 20, 2009, Hong Kong.

[10] Mohamed Ibrahim and Moustafa Youssef," CellSense: An Accurate Energy-Efficient GSM Positioning System“, Ons On Vehicular Technology, Vol. 61, NO. 1, (C) 2011 IEEE.

[11] Bashir Shalaik and Adam Winstanley," Delivering RealTime Bus Tracking Information on Mobile Devices", J.J. Park, L.T. Yang, and C. Lee (Eds.): FutureTech 2011, Part II, CCIS 185, pp. 139-147, (C) Springer-Verlag Berlin Heidelberg 2011.

[12] Mohammad A. Al-Khedher," Hybrid GPS-GSM Localization of Automobile Tracking System", International Journal of Computer Science \& Information Technology (IJCSIT) Vol 3, No 6, Dec 2011.

[13] Iman M. Almomani, Nour Y. Alkhalil, Enas M. Ahmad, Rania M. Jodeh," Ubiquitous GPS Vehicle Tracking and Management System", 2011 IEEE Jordan Conference on
Applied Electrical Engineering and Computing Technologies (AEECT), 978-1-4577-1084-1/11/\$26.00 (C)2011 IEEE.

[14] Xianyong Feng, Karen L. Butler-Purry and Takis Zourntos," Multi-Agent System-Based Real-Time Load Management for All-Electric Ship Power Systems in DC Zone Level”, 0885-8950/\$31.00 @ 2012 IEEE.

[15] Montaser N. Ramadan, Mohammad A. Al-Khedher and Sharaf A. Al-Kheder," Intelligent Anti-Theft and Tracking System for Automobiles “,International Journal of Machine Learning and Computing, Vol. 2, No. 1, February 2012.

[16] Saed Tarapiah, Shadi Atalla and Rajaa AbuHania," Smart On-Board Transportation Management System Using GPS/GSM/GPRS Technologies to Reduce Traffic Violation in Developing Countries “, International Journal of Digital Information and Wireless Communications (IJDIWC) 3(4): 96-105, ISSN: 2225658X, 2013.

[17] A. Al-Mazloum, E. Omer, M. F. A. Abdullah," GPS and SMS-Based Child Tracking System Using Smart Phone", World Academy of Science, Engineering and Technology, International Journal of Electrical, Electronic Science and Engineering Vol:7 No:2, 2013.

[18] Google Map APIs. [Online] Available at https://developers.google.com/maps/documentation/webs ervices/ [Accessed: 25 Nov 2013]. 